Hard X-ray techniques suitable for polymer experiments

This article has been downloaded from IOPscience. Please scroll down to see the full text article.

2010 IOP Conf. Ser.: Mater. Sci. Eng. 14012001

(http://iopscience.iop.org/1757-899X/14/1/012001)

View the table of contents for this issue, or go to the journal homepage for more

Download details:

IP Address: 134.58.253.57

The article was downloaded on 18/01/2011 at 12:06

Please note that terms and conditions apply. 


\title{
Hard X-ray techniques suitable for polymer experiments
}

\author{
W. Bras, H. Goossens, B. Goderis \\ Netherlands Organisation for Scientific Research (NWO) \\ DUBBLE@ESRF, BP 220, F38043 Grenoble Cedex, France \\ Department of Chemical Engineering and Chemistry, Eindhoven University of \\ Technology, P.O. Box 513, 5600 MB Eindhoven, The Netherlands \\ Molecular and Nanomaterials, Chemistry Department, Catholic University of Leuven, \\ Celestijnenlaan 200F, Belgium \\ corresponding author Wim.Bras@esrf.fr
}

\begin{abstract}
Polymers have been studied since 1979 with $8-12 \mathrm{keV}$ synchrotron radiation X-ray scattering methods and the number and sophistication of the experiments have rapidly grown ever since. More recently, new experimental techniques have been developed that use softer or harder Xrays in less conventional ways. This article provides a brief overview of the possibilities of hard X-ray techniques and indicates some areas that might gain from further developments.
\end{abstract}

\section{Introduction}

The number of synchrotron radiation (SR) sources worldwide is still increasing as many of the older sources are being phased out. In most of these laboratories at least some beam lines are being used for polymer and soft condensed matter science. In some rare cases these beam lines are fully dedicated to this subject but in most instances the situation is that the beam line is dedicated to a particular experimental technique and shared by a very mixed user community of which polymer researchers are only a subset, sometimes even only a small subset.

Below, we make a distinction between 'hard' and 'soft' X-rays. The demarcation point between these two regimes is set somewhat arbitrarily at a photon energy of about $5 \mathrm{keV}$, not for any physical reason but for a very practical one. Indeed, below $5 \mathrm{keV}$ the penetration power of $\mathrm{X}$-rays decreases rapidly and beam lines can no longer be equipped with Be-windows for example, since these would absorb the largest part of the photons. Therefore, experiments that rely on the softer energies must be carried out in a vacuum on beam lines equipped with differential pumping systems which severely limits the possibilities for on line experiments. 
The hard SR X-ray regime has already been utilized for several decades by polymer researchers and the main techniques, - small- and wide-angle scattering and diffraction - are well established. Technical developments in X-ray optics and SR sources during the last decade have led to smaller and more powerful beams so that SAXS and WAXS can now also be carried out with sub-micron sized samples. However, the technique remains virtually the same. Similar arguments can be given for experiments where the sample is illuminated by the X-ray beam under grazing incidence, rendering surface, or near surface, information. Since this group of techniques is now well established, it is possible to give a brief historical and technical overview. The situation is different for the soft X-ray regime where several of the available techniques have only recently become available for polymer science.

In studies of polymer structure and morphology X-ray scattering experiments have traditionally played an important role. Already in the classical text book of Guinier and Fournet from 1955 a whole chapter is dedicated to the application from SAXS to polymers, whilst before that many polymeric systems, either biological or synthetic in origin were studied with WAXS ${ }^{1}$. The role that X-ray scattering experiments play in polymer research is undiminished. Although the majority of work is performed in academic or industrial laboratories with conventional X-ray sources, SR beam lines have had an increasingly important role in the last two decades. One of the main reasons why synchrotron research has not reached the predominant position in polymer morphology and structure research - as it has for instance in the case of protein crystallography - is probably the lack of available beam lines. Nearly every SR laboratory nowadays has at least one X-ray scattering beam line, but this is by far not sufficient to cover the potential demand.

It is undeniable that polymer X-ray scattering has benefited from the availability of the intense $\mathrm{X}$-ray beams that SR sources can provide as illustrated by the hundreds of publications appearing each year. To our knowledge, the first record of a scattering experiment on polymers using SR appeared in $1979^{2}$. Since then, many experiments have been performed with an ever increasing degree of sophistication. Whilst time-resolved experiments were very difficult using a conventional X-ray generator they became routinely possible with synchrotron beam lines. The consequence was that the requirements for sample environment control also increased in importance. In the early years, it was already considered to be a luxury to be able to vary the temperature of a sample, in contrast to the Xray generator experiments where one was limited to fixed temperatures. Nowadays, it is feasible to build complete polymer processing installations on beam lines. Despite this, a large part of the experiments taking place on synchrotron beam lines involves still a rather straightforward variation in temperature in order to determine for instance a phase diagram or follow the crystallization process of semi-crystalline materials under quiescent conditions. 


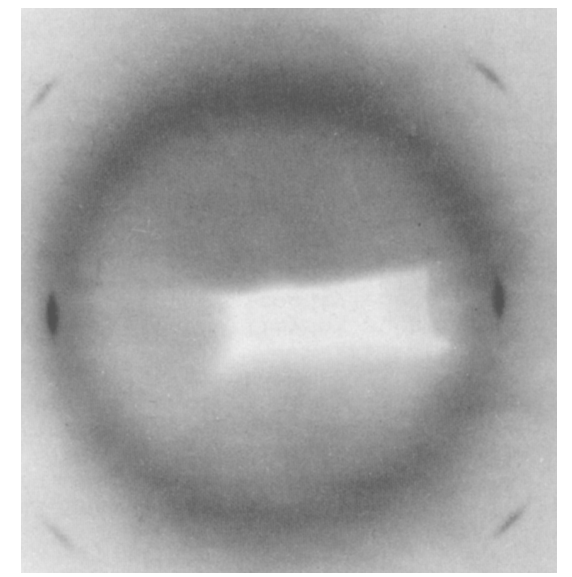

Figure 1

Probably the first published two-dimensional scattering pattern of a polymer obtained with synchrotron radiation on the X33 beam line of the EMBL at Hamburg and recorded on Osray T4 Xray film. (Data courtesy of M.H.J. Koch). The corresponding time-resolved experiments on the deformation-induced crystallization kinetics of polyisobutylene were recorded with a linear position sensitive gas detector with delay line readout built by A. Gabriel. (Figure reproduced from (2))

It is, however, interesting to note that over the years there has been a shift of emphasis in the X-ray scattering techniques used, which is well illustrated by the classical book on polymer scattering by Alexander ${ }^{3}$ where the largest part of the text is dedicated to wide-angle scattering and diffraction techniques while small-angle scattering only plays a minor role. At present, there are hardly any synchrotron beam lines that offer WAXS as a stand-alone tool as the powder diffraction beam lines, which would be an overkill for polymer systems, are mostly dedicated to high resolution studies, and the bulk of the work is performed on SAXS or combined SAXS/WAXS beam lines.

The first SAXS synchrotron beam line was designed for fiber diffraction, in particular for the study of muscle contraction. This took place at the Hamburg synchrotron ${ }^{4}$. This was later followed by a SAXS beam line dedicated to polymer research which was initiated by Prof. Zachmann of Hamburg University $^{5}$. In 1998, approximately 24 SAXS beam lines were operating ${ }^{6}$. In 1988, the first beam line completely dedicated to combined SAXS/WAXS experiments, but not necessarily limited to polymer research, was opened in Daresbury ${ }^{7}$. Worldwide, there are now more synchrotrons operating than in 1998 and the number of dedicated or partially dedicated SAXS/WAXS beam lines has increased so much that we shall not try to make an inventory here.

The advantages of using SR in X-ray scattering experiments are clear. Compared to a laboratory set-up one has in general two orders of magnitude more intensity and the divergence of the X-ray beam is much lower. This not only translates into shorter exposure times but also allows the utilization of more complicated sample environments like shear cells, reaction injection molding (RIM) machines, superconducting magnets and pressure cells. It is also possible to obtain a better low-angle resolution even with the conventional transmission SAXS configuration.

Classical research areas where synchrotron SAXS and SAXS/WAXS have been, and still are, applied are for instance phase diagram mapping ${ }^{8,9}$ and crystallization studies ${ }^{10,11}$. In principle, there is no reason why phase diagram mapping could not be done on conventional X-ray instruments but the main advantage here clearly lies in the possibility to obtain a fairly complete map in a relatively short time. Mapping of the phase diagrams of a polyisoprene-polystyrene diblock copolymer, for example, 
can be done in a single night shift on a second generation synchrotron (see figure 2) whereas in a laboratory situation a similar set of measurements would take several weeks. The reasons for using SR are even more obvious in crystallization studies. The duration of the time frames in which the data is obtained must be matched to the crystallization rate of the polymer. In a classical temperature quench experiment with a relatively high undercooling of the sample the development rate can be so fast that subsecond time-resolution is required. With conventional generators it is still not possible to obtain acceptable, analyzable and interpretable data sets with such fast data acquisition rates ${ }^{12}$.

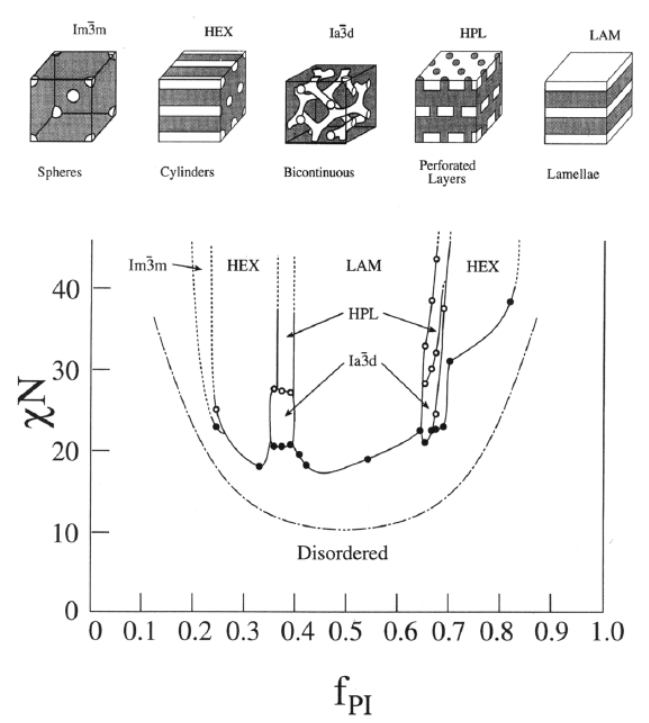

Figure 2

Polyisoprene-polystyrene diblock copolymer compositional phase diagram. The abscissa is the fraction of polyisoprene and the ordinate the product of the Flory-Huggins parameter multiplied by the degree of polymerization. Such a phase diagram can be obtained by SAXS with a SR beamline in a single night shift ${ }^{9}$ whereas this could well take weeks on a conventional instrument. (reprinted with permission from (9))

A debate about the crystallization pathway of semi-crystalline polymers flared up around 1998 where one tried to decide whether there was a conventional nucleation and growth mechanism or intermediate states that possibly could be described by a spinodal decomposition type of mechanism ${ }^{10}$ 11, 13. This required combined SAXS/WAXS experiments in which the sequence in which changes could be observed in the long and short length scale were of importance. Independently of the outcome of this debate this highlighted not only the shortcomings of the detector systems in use on different beam lines but also that SAXS was intrinsically more sensitive to changes than WAXS ${ }^{14}$. Even with equal sensitivity of the SAXS and WAXS detector one would always find a signature excess SAXS intensity before one would discern crystalline peaks. This could be the case as well in some of the non-polymer crystallization experiments, which presently are abundant in the literature and where one finds an amorphous phase before one observes the crystalline peaks. Scherrer and thermal broadening as well as detector issues and the intrinsic sensitivity difference might play a role in some cases in preventing the detection of the peaks above the background.

Although a relatively high X-ray intensity is required for time-resolved experiments, one should not get overexcited and perform too long term experiments on a single sample, or use a very high intensity beam for shorter periods, simply because radiation damage cannot be avoided even not at a second generation synchrotron source or a bending magnet. A series of SAXS/WAXS experiments at 
$8 \mathrm{keV}$, with around $10^{10}$ photons/sec, for about 8 hours of continued irradiation on a single ethylene copolymer proved this point. For non-exposed samples DSC experiments demonstrated that any thermal history could be wiped out by cooling from a high temperature melt, i.e. $200^{\circ} \mathrm{C}$. The standard crystallization peak temperature could easily be reproduced. However, when such thermal resets were run at regular time intervals during the 8 hour experiment an unexpected result was found. The temperature at the peak in the first derivative of the SAXS total scattered intensity (the invariant) in these runs was expected to roughly compare with the standard crystallization peak temperature in DSC ${ }^{15}$ Unexpectedly, these SAXS based crystallization peak temperatures were found to shift to lower temperatures with time rather than to the standard temperature, demonstrating that in scattering, the sample history could not efficiently be wiped out (see Figure 3, left panel). Irradiated and nonirradiated fragments of the sample were recovered after the 8 hour experiment and analyzed by DSC and FTIR. It turned out that the irradiated fragment started cross-linking already very early during the experiment whereas the non-irradiated parts were left intact (Figure 3, right panel). This experience only found a place in the 1995 Hasylab annual user reports but is probably worth sharing, thereby mentioning as a rule of thumb that irradiation times - even at a bending magnet - should be limited to about 30 min for polyolefins and likely for many other systems or that fast shutters should be used in longer term experiments to reduce the actual irradiation time ${ }^{16}$
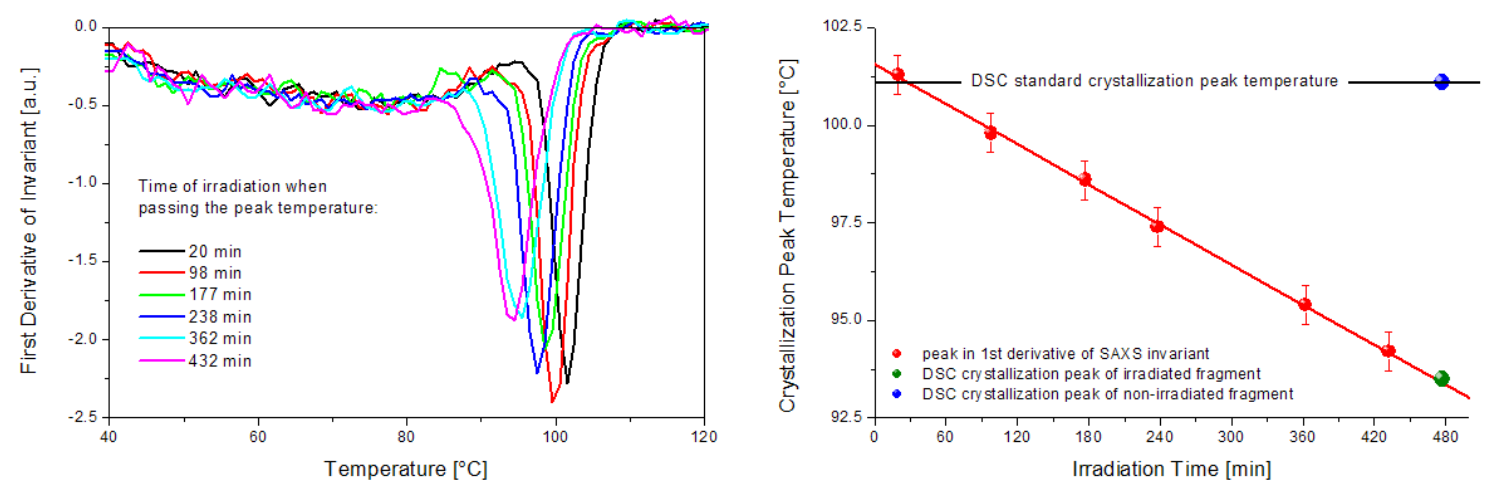

Figure 3: Left panel: First derivative of total SAXS intensity (invariant) during cooling an ethylene copolymer at different irradiation times $(8 \mathrm{keV})$; Right panel: SAXS invariant based crystallization peaks (red markers) together with the crystallization peaks from DSC of material that was in the beam for 8 hours (green marker) and of material that was in the same sample holder but outside the beam (blue marker).

The low divergence of the synchrotron X-ray beams makes it possible to use large sample environments in SAXS experiments. Where when using conventional generators the beam would already have diverged so far that only a small fraction of the original beam would still reach the sample with synchrotron beams it is posssible to travel several meters without loosing too much intensity under the condition that the X-ray path is evacuated. An example of a large sample environment is for instance a superconducting magnet which can be used for sample alignment and dynamic reorientation studies ${ }^{17,18}$. The path length through the magnet is about $400 \mathrm{~mm}$. This is roughly on par with the focal distance of X-ray optics generally used on X-ray generator sets. This would then lead to the sample being placed in the focal spot instead of the detector and thus would bring considerable blurring and loss of low angle resolution, once the beam has traveled towards the detector.

The low divergence and high-intensity X-rays have also allowed the installation of equipment suitable for complementary experiments. In this case, one can think about a whole array of different shear devices ranging from parallel plate devices to Couette cells. As an example of why SR beams 
are particularly useful for the Couette geometry one should remember that 'shooting' the beam through the Couette gap is a lot easier with a small beam than with that of a conventional generator. The fact that the beam has to travel through the materials of the Couette cylinders at a rather oblique angle means that a lot of radiation will be absorbed. This is one of the reasons why this type of experiment was long confined to neutron scattering beam lines. In contrast to popular belief, the intensity of the direct beam is of no importance here, since a strong absorption is also accompanied by strong multiple scattering and a concomitant deterioration of the quality of the scattering patterns. The saving grace at third generation SR beam lines is that they quite often are tuneable in energy. By increasing the energy of the photon beam one reduces the multiple scattering component of the detected pattern considerably. In such cases it is generally also beneficial to increase the sample thickness at least to match it as much as possible to the optimum thickness for the specific photon energy. These issues are not only relevant to Couette cells or other shearing devices but also to e.g. diamond anvil cells for high pressure work.

Most of the beam lines used for polymer X-ray scattering on the first two generations of SR sources had a fixed wavelength due to the requirement to generate sufficient X-ray flux on the sample and the optical elements that could provide that flux ${ }^{2,19}$. The photon energy was in general not far removed from the familiar $\mathrm{Cu}-\mathrm{K} \alpha$ line used in conventional generators which in turn was not far from the critical energy for most of these storage rings, thus assuring the highest possible flux whilst still keeping the potential user on familiar ground. This photon energy leaves one with an optimum sample thickness around 2-3 $\mathrm{mm}$ for the average polymer sample which is composed predominantly of $\mathrm{C}, \mathrm{H}$ and $\mathrm{O}$. The combination of an optimized flux and an appropriate sample thickness allowed timeresolved experiments even with the relatively inefficient gas-filled wire chambers which were the main stay of detectors in the early years ${ }^{20,21}$.

With the present generation of hard X-ray SR beam lines the X-ray flux is so large that one can afford to loose some of the photons by using a double crystal monochromator which will reduce the flux somewhat due to the smaller energy bandwidth transmitted. Even the requirement to use a sample thickness which is close to the optimum sample thickness can be relaxed by using thinner samples. In fact, the flux is nowadays often so high that radiation damage to the samples has to be taken into consideration, exacerbating the problems found at second generation synchrotron sources, as mentioned above. The saving grace here is that finally efficient Si-based detectors have come onto the market which are capable of counting single photons ${ }^{22}$. The earlier generation of integrating Si-based CCD detectors were detrimental for the data quality in the intermediate scattering range due to the rapid fall off in intensity at medium scattering angles which meant that even with the lowest read out speeds the electronic noise was already rapidly of the same order as the scattering signal unless one used radiation doses so high that one had to fear for radiation damage and saturation in the low angle part of the pattern .

One of the important remaining bottlenecks in X-ray diffraction and scattering is the lack of generally accepted software to analyze SAXS and fiber diffraction data. This situation is aggravated when dealing with time-resolved data where the data volume can be rather large and even with modern computers this might be a non trivial task. The problem of data set size can sometimes be handled by a careful assessment of the required spatial resolution of the detectors for a specific system. One has become so accustomed to the high spatial resolution offered by for instance CCD detectors that one rarely considers whether this resolution is really required or even sensible. For instance when dealing with beam sizes of around 300 micron it rarely makes sense to collect data with a detector with a pixel size of 100 microns. The data could easily be binned in larger blocks when the detector allows such. For a popular workhorse detector like the MAR SX165 the difference is a factor 16 in frame size. However, even with reasonable sized data sets the problem remains that there are is a lack of data analysis software packages suitable for the analysis of a variety of problems. Widely used programs 
are presently only available for dilute monodisperse solutions. For other problems, especially where there is partial orientation or even full fibre symmetry, one in general has to rely on home developed software routines. Some attempts to develop analysis software suitable for a larger number of scattering problems and capable of handling time-resolved data in an intelligent way, have been made $^{23,24}$ and elaborate packages for composite polymer fibres are also available ${ }^{25}$. However, so far the lack of easily available, robust and inexpensive software is a major obstacle in the polymer X-ray scattering field.

Besides data analysis, data reduction can also be problematic. Here, we define as data reduction the data manipulations that are required to obtain the corrected scattering curves and as analysis the manipulations that are required to obtain the structural parameters of the samples. Data reduction software is often influenced by the local beam line hardware and detectors and in most cases have some simple options for preliminary analysis of the easiest to calculate parameters. Whereas 2D SAXS detectors are mostly put perpendicular to the direct beam, in a combined SAXS/WAXD experiment the WAXD detectors are placed close to bulky equipment and most times not in the optimal geometric position. However, few data reduction packages are capable of dealing with all corrections required for data collected with detectors placed under such awkward detector positions. Recently, software has been developed to cope with the angular calibration and averaging of such, at times, heavily distorted 2D powder patterns ${ }^{26}$.

As mentioned above the possibilities to utilize more complex sample environments and also combine the X-ray scattering experiments with complementary on-line techniques is another major advantage of the use of SR. A whole variety of shear, deformation and chemical cells is nowadays available and a considerable number of techniques can be combined. Examples are: Raman spectroscopy, light scattering, thermal analysis, optical microscopy, dielectric spectroscopy, X-ray spectroscopy and many others. In fact, compared to the situation in 1998 when this subject was still fairly limited ${ }^{6}$, there are that many sample environments and combinations implemented over the last decade that it is next to impossible to give an overview here. It is good to remind ourselves occasionally that such experiments were already attempted more than 20 years ago by the groups of H.G. Zachmann in Hamburg and H. Reynaers in Leuven.

The first conference dedicated to the use of SR in polymer science (SRPS) was organized in 1995 in Hamburg by the late Prof H.G. Zachmann, the pioneer in this field, and was heavily dominated by results of X-ray scattering and diffraction using hard X-rays. The situation was very similar in the subsequent two conferences, Sheffield (2002) and Kyoto (2006). However, it is clear that in the last few years some less conventional SR techniques, mainly using softer X-rays, have become available to the polymer research community. Therefore, it was decided that a larger part of the SRPS 4 (Rolduc, the Netherlands, 2009) program would be reserved for these less frequently used techniques. In this contribution we give a brief overview of the historical development and present status of the more conventional techniques using hard X-rays. The organizers have approached several authors working with softer X-rays and managed to convince them to provide a contribution in order to give a more detailed overview of what is presently feasible

\section{Modern developments}

The hard X-ray scattering and diffraction experiments have in time increased in sophistication. Initially due to the increase in X-ray flux but over the last decade more through the fact that a virtual $\mathrm{X}$-ray flux threshold has been crossed. This has allowed for the construction of a large array of high to super high flux X-ray scattering beam lines with tunable energies. This, and the advent of more efficient detectors, in turn has allowed the introduction of more complex sample environments in 
which fewer concessions have to be made to the X-ray instrumentation. Increased flux and X-ray beam collimation will still allow some marginal improvements in conventional transmission scattering experiments but the emphasis in instrumentation developments has definitely shifted to the development of new sample environments and technique combinations.

It is foreseeable that the role of conventional SAXS/WAXS experiments will not diminish much . A large body of work is still dedicated to determine and improve processing conditions for polyolefins for which the scattering experiments are indispensable. See figure 4 . These are still by far the most used polymers, as measured by annual production. As such one can expect the demand for SAXS/WAXS beam lines to remain high. This will certainly be augmented by the rapidly growing interest in bio-based polymers and polymers for electronics which also require X-ray scattering for morphological characterisation.

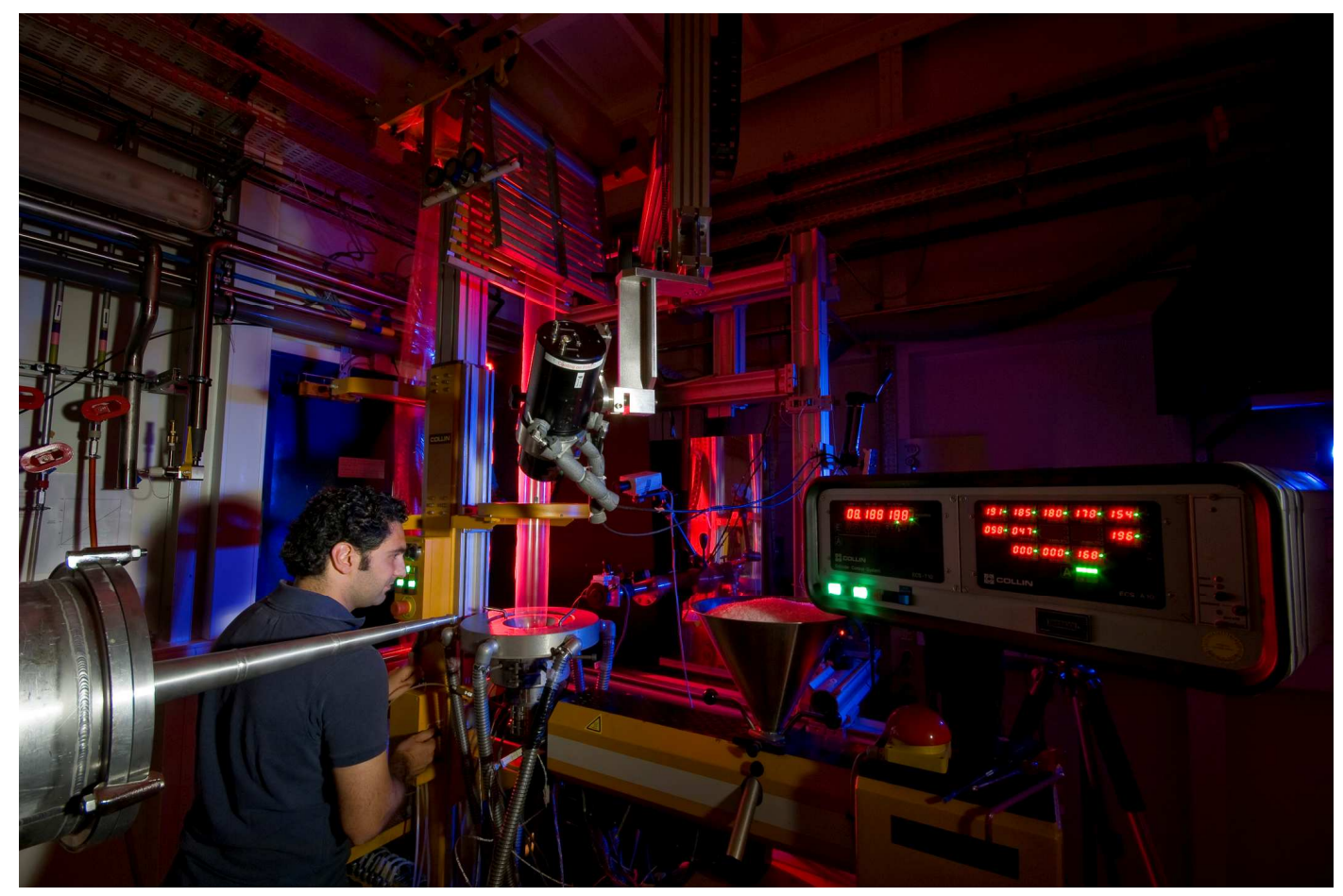

Figure 4

On-line polymer film blowing of polyethylene. This type of experiment emphasizes the points that in the first place the sample environments are getting more complicated and in the second place research on polyolefins is still alive and well. Experiments on BM26B at the ESRF in a collaboration between Genoa and Eindhoven Universities.

A logical development on third generation SR sources is to implement optics that can achieve a small focal spot size. The X-ray sources and optics have now been developed so far that submicron beams have become possible. Obviously radiation damage issues should not be underestimated in this case, since in order to create a diffraction pattern with sufficient statistics one requires a certain number of photon-sample interactions. For the detectors it makes no difference if these interactions have been generated in a small or large sample volume but this is clearly different for the sample. This makes the microfocus diffraction/scattering technique particularly suitable for spatially scanning samples in order to determine the local structure variations that would be averaged with a larger beamsize. An early polymer research example was the determination of the lamellar orientations in a poly-3-hydroxybutyrate spherulite ${ }^{27}$. For time-resolved experiments there is the problem that one 
requires to illuminate the sample spot of interest for the duration relevant for the development of structure, although not necessarily continuously. This raises again the spectre of radiation damage but in some cases it is possible to design the experiment in such a way that this problem can be overcome. A spectacular example is given by Riekel and Volrath where a spider was used as an on-line extruder $^{28}$. By varying the distance between the spiders spinning spigot and the X-ray beam one can translate the crystallization time into a physical distance with new, undamaged material continuously being exposed to the beam. On-line melt spinning was already carried out in 1993 in Hamburg ${ }^{29}$ but the properties of a third generation SR source was required to be able to carry out such experiments with very thin natural polymer fibres. Another beautiful example of the application of microfocus is the determination of the lamellar twist in semi-crystalline polymers which is being discussed in these conference proceeding ${ }^{30,31}$. See figure 5 . A fairly recent overview of developments using microfocus in the field of polymer and biopolymer research can be found in Riekel and Davies ${ }^{32}$.
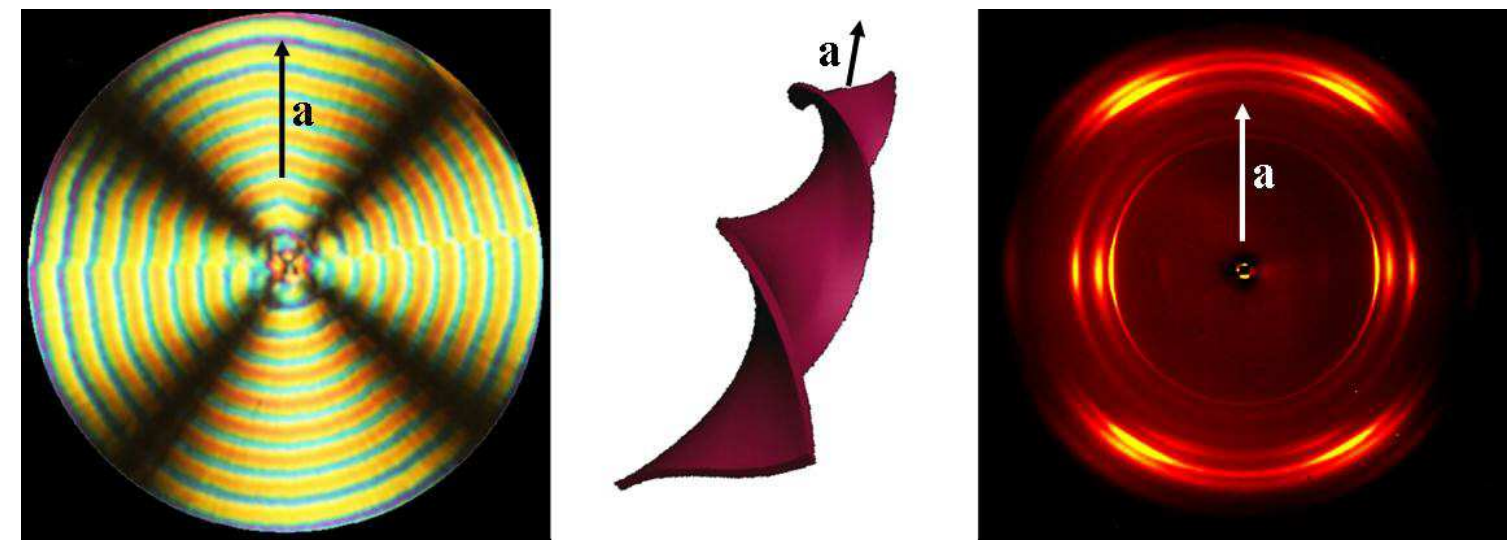

Figure 5

Optical micrograph of a banded spherulite of polytrimethylene terephthalate, PTT, melt-crystallized at $170^{\circ} \mathrm{C}$ (left panel). The band spacing is $23.5 \mu \mathrm{m}$. Schematic drawing of a twisted lamellar crystal (middle), which is the main constitutive element of the banded spherulite morphology. The axis of twist corresponds the to the crystallographic a-axis of the PTT unit cell. X-ray pattern obtained by summing up an array of micro-focus patterns measured during a radial scan across the PTT spherulite (right). The vertical axis of the pattern corresponds to the fast crystal growth direction, i.e. a. (Figures courtesy D.A. Ivanov and M. Rosenthal)

Another technique that has become more popular in recent years is Grazing Incidence SAXS. This technique was first used in 1989 using a rotating anode and hard condensed matter samples ${ }^{33}$, but it was soon recognized that this was a technique that could really benefit from the superior beam quality of SR sources. The introduction of this technique to polymer samples occurred some years later and has taken off exponentially in the last years. This evolution reflects less the advances made in beam line instrumentation than those in polymer synthesis and processing which allow to produce very well defined nanometer-sized structured materials for instance by self assembly processes as is illustrated in figure 6. Since the experimental requirements are not much different than for more conventional transmission, many SAXS instruments are equipped to perform both techniques. When the angle of the sample surface with respect to the direct beam is very shallow the X-ray beam only penetrates the sample to a small distance below the surface thereby probing only a thin layer. The highly collimated $\mathrm{X}$-ray beams that can be generated at SR sources are in this case very useful since the 'footprint' of the beam on the sample in the grazing incidence geometry can be smaller than on conventional sources. 

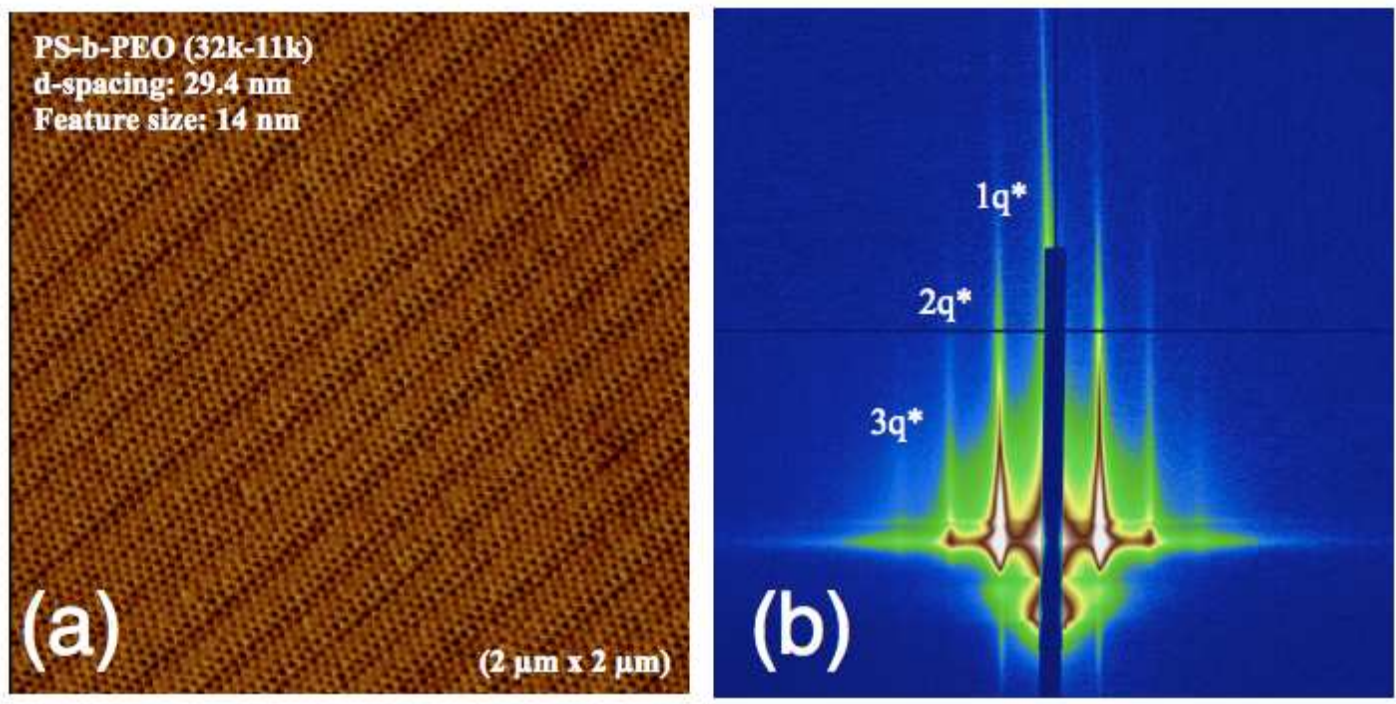

Figure 6

GISAXS results on a self assembled PS-PEO block copolymer which forms a macroscopic 10Terabit-per-Square- Inch Arrays storage medium. Panel a shows an SEM picture and panel $\mathrm{b}$ shows the corresponding scattering pattern. (Data obtained on ALS beamline 7.3.3. Figure courtesy of Tom P. Russell and Alex Hexemer) ${ }^{34}$

The use of hard X-rays for scattering or diffraction purposes experiments has gained wide acceptance through the years not in the least due to the fact that with conventional generators these techniques also can be carried out and synchrotron-based experiments are a logical extension of experiments that can not be done or take too much time at home. No basic new experimental skills have to be learned. This is in contrast to techniques that require either soft or very hard X-ray or a continuous spectrum. For these experiments there hardly exists a tradition in non-SR based laboratories due to there being, realistically, no sources available other than SR sources. Therefore, the user community that wants to use these techniques in general has to overcome the double entropy barrier of having to travel to a large facility as well as having to acquire the expertise required to carry out the new experiments. As a consequence, the user group practicing these techniques is not very large. However, sometimes these methods provide unique information, not obtainable in any other way.

One of the fields where harder X-rays are required due to their higher penetration power is X-ray tomography. This technique renders three-dimensional structures. The underlying principle is that one obtains many transmission projections as a function of rotation angle with respect to the direct beam. In order to be able to penetrate samples of several millimeters thick it is required to use higher photon energy (> $20 \mathrm{keV})$ and hence the role of $\mathrm{SR}^{35,36}$.

In figure 7 a tomograph of a piece of polyurethane foam is shown. The sample dimensions were about $4 \times 4 \times 4 \mathrm{~mm}^{3}$. A tomograph like this can be obtained in several minutes which in principle makes the technique suitable for slow time-resolved experiments. However, one should realize that the required computing effort for a time-resolved experiment would be substantial. 


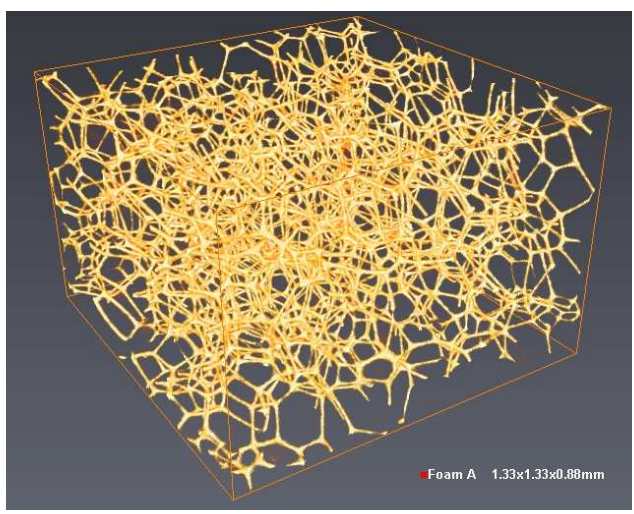

Figure 7

Tomography results on a polyurethane foam. The data was obtained on beam line 8.3.2 at the ALS on a block of $4 \times 4 \times 4 \mathrm{~mm}^{3}$. Data collection was performed in several minutes but data reduction was considerably longer. With better detectors these experiments could be performed in a time-resolved way. Data courtesy of Alistair McDowell (ALS)

In order to progress from solely the production of good looking pictures to real information a statistical analysis of parameters like strut length and pore volume size distribution must be performed. This is also a non-trivial task but several image recognition software packages exist. Such statistical information can be correlated with the macroscopic properties as shown with foams that were stepwise compressed.

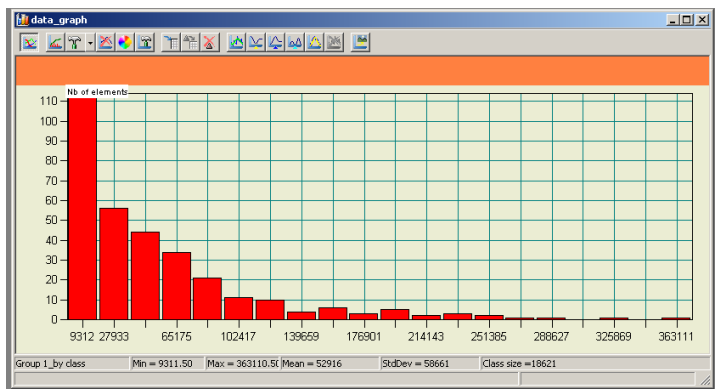

Figure 8

Pore Volume distribution derived from the data shown in figure 7. The time required to extract this information from the experimental data is considerable.

In figure 8 derived data from the polyurethane foam are shown. This and other parameters, like strut length, can be used for engineering calculations relating this microscopic data to measurable macroscopic parameters.

Hard X-ray spectroscopy (EXAFS/XANES) is not often used in polymer studies due to the absence of suitable absorption edges. Above photon energies of $5 \mathrm{keV}$ there are no edges for $\mathrm{C}, \mathrm{H}, \mathrm{O}$ and $\mathrm{N}$. These experiments only yield useful results in a small niche for materials like ionomers which contain metallic elements ${ }^{37-39}$. The situation is obviously different when one also considers nanocomposite systems, like clay-polymer composite materials, in which one of the components contains metallic elements $^{40}$. The situation is also markedly different for photon energies below $5 \mathrm{keV}$ and in recent years several groups have started using techniques that utilize this energy range to obtain spectroscopic information. These experiments are treated in several contributions to the proceedings of this conference. 


\section{Conclusions}

Polymer research is now widely spread at SR facilities. The largest part is still hard X-ray scattering which can be viewed as an extension of work on conventional sources. This 'domination' is unlikely to change but several new techniques have evolved during the last decade.

There are two main problems with the conventional scattering and diffraction methods. First there is a lack of suitable beam lines which makes beam time scarce. There is no real justification for this shortage of beam lines, since the demand from the user community is there. However, since SAXS and WAXS beam lines are usually classified as 'sample characterization beam lines', they are often perceived as producing less cutting edge results and are therefore less popular with funding agencies and the management of SR laboratory facilities. This is a fate they share with for instance powder diffraction and X-ray spectroscopy beam lines. Ironically, however, these type of beam lines are often the most productive. Here the community could help by a better "marketing" of their results, which often requires no more than a pretty picture.

The second problem that the community faces is the lack of easily accessible data analysis software and that this tends to result in a backlog of unanalysed data. The most frequently used software packages are mainly geared towards the simplest systems such as monodisperse and dilute samples and are not adequate for polydisperse, concentrated, disordered and partially oriented samples which are most often encountered in materials science. Availability of appropriate software packages would not only speed up data analysis but in all likelihood also improve its quality.

It is, however, encouraging to see that new SR-based techniques are becoming available to the polymer research community. These offer new characterization tools with a chemical specificity that the hard X-ray scattering and imaging techniques lack.

We hope that in the proceedings of this conference one can not only discover the possibilities that the more traditional scattering techniques can presently offer at the moment but that also a broad overview of the newly developing techniques is given.

\section{Acknowledgements}

Michel Koch is gratefully acknowledged for a reading of the manuscript.

\section{References}

1. Guinier, A.; Fournet, G., Small Angle Scattering of X-rays. John Wiley \& Sons: 1955.

2. $\quad$ Koch, M. J. H.; Bordas, J.; Schola, E.; Broecker, H. C., KINETIC STUDY OF THE

CRYSTALLIZATION OF STRETCHED POLYISOBUTYLENE USING SYNCHROTRON

RADIATION. Polymer Bulletin 1979, 1 (10), 709-714.

3. $\quad$ Alexander, L. E., X-ray diffraction methods in polymer science. Wiley: 1969.

4. Rosenbau.G; Holmes, K. C.; Witz, J., SYNCHROTRON RADIATION AS A SOURCE

FOR X-RAY DIFFRACTION. Nature 1971, 230 (5294), 434-\&.

5. Bark, M.; Zachmann, H. G.; Alamo, R.; Mandelkern, L., Investigations Of The

Crystallization Of Polyethylene By Means Of Simultaneous Small-Angle And Wide-Angle X-Ray-

Scattering. Makromolekulare Chemie-Macromolecular Chemistry And Physics 1992, 193 (9), 2363 2377.

6. Bras, W.; Ryan, A. J., Sample environments and techniques combined with Small Angle Xray Scattering. Advances in Colloid and Interface Science 1998, 75 (1), 1-43. 
7. Bras, W.; Derbyshire, G. E.; Ryan, A. J.; Mant, G. R.; Felton, A.; Lewis, R. A.; Hall, C. J.; Greaves, G. N., Simultaneous Time Resolved Saxs and Waxs Experiments Using Synchrotron Radiation. Nuclear Instruments \& Methods in Physics Research Section a-Accelerators Spectrometers Detectors and Associated Equipment 1993, 326 (3), 587-591.

8. $\quad$ Forster, S.; Khandpur, A. K.; Zhao, J.; Bates, F. S.; Hamley, I. W.; Ryan, A. J.; Bras, W., Complex Phase-Behavior of Polyisoprene-Polystyrene Diblock Copolymers near the Order-Disorder Transition. Macromolecules 1994, 27 (23), 6922-6935.

9. $\quad$ Khandpur, A. K.; Forster, S.; Bates, F. S.; Hamley, I. W.; Ryan, A. J.; Bras, W.; Almdal, K.; Mortensen, K., Polyisoprene-polystyrene diblock copolymer phase diagram near the order-disorder transition. Macromolecules 1995, 28 (26), 8796-8806.

10. Terrill, N. J.; Fairclough, P. A.; Towns-Andrews, E.; Komanschek, B. U.; Young, R. J.; Ryan, A. J., Density fluctuations: the nucleation event in isotactic polypropylene crystallization. Polymer 1998, 39 (11), 2381-2385.

11. Wang, Z. G.; Hsiao, B. S.; Sirota, E. B.; Srinivas, S., A simultaneous small- and wide-angle $\mathrm{X}$-ray scattering study of the early stages of melt crystallization in polyethylene. Polymer 2000, 41 (25), 8825-8832.

12. Heeley, E. L.; Fernyhough, C. M.; Graham, R. S.; Olmsted, P. D.; Inkson, N. J.; Embery, J.; Groves, D. J.; McLeish, T. C. B.; Morgovan, A. C.; Meneau, F.; Bras, W.; Ryan, A. J., Shear-induced crystallization in blends of model linear and long-chain branched hydrogenated polybutadienes.

Macromolecules 2006, 39 (15), 5058-5071.

13. Strobl, G., From the melt via mesomorphic and granular crystalline layers to lamellar crystallites: A major route followed in polymer crystallization? Eur. Phys. J. E 2000, 3 (2), 165-183.

14. Bras, W.; Dolbnya, I. P.; Detollenaere, D.; van Tol, R.; Malfois, M.; Greaves, G. N.; Ryan, A. J.; Heeley, E., Recent experiments on a combined small-angle/wide-angle X-ray scattering beam line at the ESRF. Journal of Applied Crystallography 2003, 36, 791-794.

15. Bras, W.; Derbyshire, G. E.; Devine, A.; Clark, S. M.; Cooke, J.; Komanschek, B. E.; Ryan, A. J., The Combination of Thermal-Analysis and Time-Resolved X-Ray Techniques - a Powerful Method for Materials Characterization. Journal of Applied Crystallography 1995, 28, 26-32.

16. Goderis, B.; Reynaers, H.; Scherrenberg, R.; Mathot, V. B. F.; Koch, M. H. J., Temperature reversible transitions in linear polyethylene studied by TMDSC and time-resolved, temperaturemodulated WAXD/SAXS. Macromolecules 2001, 34 (6), 1779-1787.

17. Gielen, J. C.; Wolffs, M.; Portale, G.; Bras, W.; Henze, O.; Kilbinger, A. F. M.; Feast, W. J.; Maan, J. C.; Schenning, A.; Christianen, P. C. M., Molecular Organization of Cylindrical Sexithiophene Aggregates Measured by X-ray Scattering and Magnetic Alignment. Langmuir 2009, 25 (3), 1272-1276.

18. Bras, W.; Emsley, J. W.; Levine, Y. K.; Luckhurst, G. R.; Seddon, J. M.; Timimi, B. A., Field-induced alignment of a smectic-A phase: A time-resolved x-ray diffraction investigation. Journal of Chemical Physics 2004, 121 (9), 4397-4413.

19. Lemonnier, M.; Fourme, R.; Rousseaux, F.; Kahn, R., X-ray curved crystal monochromator system at the storage ring DCI. Nuclear Instruments and Methods 1978, 152, 173-177.

20. Gabriel, A.; Dauvergne, F.; Rosenbaum, G., Linear, Circular and 2 Dimensional PositionSensitive Detectors. Nuclear Instruments \& Methods 1978, 152 (1), 191-194.

21. Bordas, J.; Koch, M. H. J.; Clout, P. N.; Dorrington, E.; Boulin, C.; Gabriel, A., A SYNCHROTRON RADIATION CAMERA AND DATA ACQUISITION-SYSTEM FOR TIME RESOLVED X-RAY-SCATTERING STUDIES. Journal of Physics E-Scientific Instruments 1980, 13 (9), 938-944.

22. Broennimann, C.; Eikenberry, E. F.; Henrich, B.; Horisberger, R.; Huelsen, G.; Pohl, E.; Schmitt, B.; Schulze-Briese, C.; Suzuki, M.; Tomizaki, T.; Toyokawa, H.; Wagner, A., The PILATUS $1 \mathrm{M}$ detector. Journal Of Synchrotron Radiation 2006, 13, 120-130.

23. Ilavsky, J.; Jemian, P. R., Irena: tool suite for modeling and analysis of small-angle scattering. Journal of Applied Crystallography 2009, 42, 347-353. 
24. Forster, S.; Apostol, L.; Bras, W., Scatter: software for the analysis of nano- and mesoscale small-angle scattering. Journal of Applied Crystallography 43, 639-646.

25. Stribeck, N.; Bayer, R.; Bosecke, P.; Camarillo, A. A., Visualisation of the structure transfer between an oriented polymer melt and the semi-crystalline state. Polymer 2005, 46 (8), 2579-2583.

26. Gommes, C. J.; Goderis, B., CONEX, a program for angular calibration and averaging of two-dimensional powder scattering patterns. Journal of Applied Crystallography 43, 352-355.

27. Mahendrasingam, A.; Martin, C.; Fuller, W.; Blundell, D. J.; Mackerron, D.; Rule, R. J.; Oldman, R. J.; Liggat, J.; Riekel, C.; Engstrom, P., MICROFOCUS X-RAY-DIFFRACTION OF SPHERULITES OF POLY-3-HYDROXYBUTYRATE. Journal Of Synchrotron Radiation 1995, 2, 308-311.

28. Riekel, C.; Vollrath, F., Spider silk fibre extrusion: combined wide- and small-angle X-ray microdiffraction experiments. International Journal of Biological Macromolecules 2001, 29 (3), 203210.

29. Cakmak, M.; Teitge, A.; Zachmann, H. G.; White, J. L., ONLINE SMALL-ANGLE AND WIDE-ANGLE X-RAY-SCATTERING STUDIES ON MELT-SPINNING POLY(VINYLIDENE FLUORIDE) TAPE USING SYNCHROTRON RADIATION. Journal Of Polymer Science Part BPolymer Physics 1993, 31 (3), 371-381.

30. Luchnikov, V. A.; Ivanov, D. A., Microbeam X-ray diffraction from twisted lamellar crystals: theory and computer simulation. Journal of Applied Crystallography 2009, 42, 673-680.

31. Ivanov, D. A.; Bar, G.; Dosiere, M.; Koch, M. H. J., A Novel View on Crystallization and Melting of Semirigid Chain Polymers: The Case of Poly (trimethylene terephthalate). Macromolecules 2008, 41 (23), 9224-9233.

32. Riekel, C.; Davies, R. J., Applications of synchrotron radiation micro-focus techniques to the study of polymer and biopolymer fibers. Current Opinion In Colloid \& Interface Science 2005, 9 (6), 396-403.

33. Levine, J. R.; Cohen, L. B.; Chung, Y. W.; Georgopoulos, P., GRAZING-INCIDENCE SMALL-ANGLE X-RAY-SCATTERING - NEW TOOL FOR STUDYING THIN-FILM GROWTH. Journal of Applied Crystallography 1989, 22, 528-532.

34. Park, S.; Lee, D. H.; Xu, J.; Kim, B.; Hong, S. W.; Jeong, U.; Xu, T.; Russell, T. P., Macroscopic 10-Terabit-per-Square- Inch Arrays from Block Copolymers with Lateral Order. Science 2009, 323 (5917), 1030-1033.

35. Elmoutaouakkil, A.; Fuchs, G.; Bergounhon, P.; Peres, R.; Peyrin, F., Three-dimensional quantitative analysis of polymer foams from synchrotron radiation x-ray microtomography. Journal of Physics D-Applied Physics 2003, 36 (10A), A37-A43.

36. Pyun, A.; Bell, J. R.; Won, K. H.; Weon, B. M.; Seol, S. K.; Je, J. H.; Macosko, C. W., Synchrotron X-ray microtomography for 3D imaging of polymer blends. Macromolecules 2007, 40 (6), 2029-2035.

37. Grady, B. P.; Floyd, J. A.; Genetti, W. B.; Vanhoorne, P.; Register, R. A., X-ray absorption spectroscopy studies of zinc-neutralized ethylene methacrylic acid ionomers. Polymer 1999, 40 (2), 283-288.

38. Grady, B. P.; Goossens, J. G. P.; Wouters, M. E. L., Morphology of zinc-neutralized maleated ethylene - Propylene copolymer ionomers: Structure of ionic aggregates as studied by X-ray absorption spectroscopy. Macromolecules 2004, 37 (23), 8585-8591.

39. Metselaar, G. A.; Schwartz, E.; de Gelder, R.; Feiters, M. C.; Nikitenko, S.; Smolentsev, G.; Yalovega, G. E.; Soldatov, A. V.; Cornelissen, J.; Rowan, A. E.; Nolte, R. J. M., X-ray spectroscopic and diffraction study of the structure of the active species in the Ni-II-catalyzed polymerization of isocyanides. Chemphyschem 2007, 8 (12), 1850-1856.

40. $\quad$ Si, M. Y.; Zaitsev, V.; Goldman, M.; Frenkel, A.; Peiffer, D. G.; Weil, E.; Sokolov, J. C.; Rafailovich, M. H., Self-extinguishing polymer/organoclay nanocomposites. Polymer Degradation and Stability 2007, 92 (1), 86-93. 